\title{
The Context-Dependency of Temporal Reference in Event Semantics
}

\author{
Fabio Pianesi ${ }^{1}$ and Achille C. Varzi ${ }^{2}$ \\ ${ }^{1}$ ITC- Istituto per la Ricerca Scientifica e Tecnologica, 38050 Povo (TN), Italy \\ pianesi@itc.it \\ ${ }^{2}$ Department of Philosophy, Columbia University, New York, NY 10027, USA \\ achille.varzi@columbia.edu
}

\begin{abstract}
Temporal reference in natural language is inherently context dependent: what counts as a moment in one context may be structurally analysed in another context, and vice versa. In this note we outline a way of accounting for this phenomenon within event-based semantics.
\end{abstract}

\section{Introduction}

It is an important characteristic of natural language that temporal relations among events can be refined at will. What counts as a moment at one level of analysis may count as extended at a deeper level:

(1) John and Mary met last week. More exactly, it was Tuesday. They met at breakfast. John was just having his first sip of coffee . . .

Even if there is a point beyond which refinement is no longer practically feasible, it seems that this is not enough to posit temporal discreteness as linguistically relevant: the underlying model of time is dense. On the other hand, natural language also permits us to discretize time at will:

(2) That's how they met: At a certain point, John asked the waiter to invite her at his table; the next moment she was sitting in front of him.

These seemingly opposite phenomena are a fundamental manifestation of the inherent context-dependency of time granularity in natural language: what counts as a moment in one context may be structurally analysed in another context, and vice versa. This is often cited as a source of difficulties for event-based semantics, where models are constructed on the basis of a definite set of events with a fixed structure. In this note we outline a way of refining such models so as to deal with these difficulties.

\section{Event Structures}

Event-based semantics originated with Davidson [3] and received algebraic formulations in Kamp [5] and Bach [1]. Such semantics are typically based on structures in

P. Bouquet, P. Brezillon, and L. Serafini (eds.), Modeling and Using Context. Proceedings of the Second International and Interdisciplinary Conference, Berlin and Heidelberg: Springer-Verlag, 1999, pp. 507-510. 
which a domain of events is associated with explicit temporal relations. In [6] we have shown that such relations can ultimately be construed from some basic ontological properties that a domain of events must arguably satisfy-specifically, mereological and topological properties. The construction is refined in [7], and in [8] it is shown to be especially liable to the sort of refinement that is requiired to account for the context-dependence exhibited by natural language. Here is a brief review of this construction.

The underlying mereotopological machinery is developed within a first-order language with identity and descriptions. The mereological and topological primitives are the parthood predicate (symbolized by ' $\mathrm{P}$ ') and the closure operator (symbolized by ' $c$ '). As axioms for these primitives we assume those of classical extensional mereology supplemented with the mereologized analogues of the standard topological axioms for closure systems. (See [2] for a full account of the theory defined by these axioms.) Additional derived notions can then be introduced by definition:
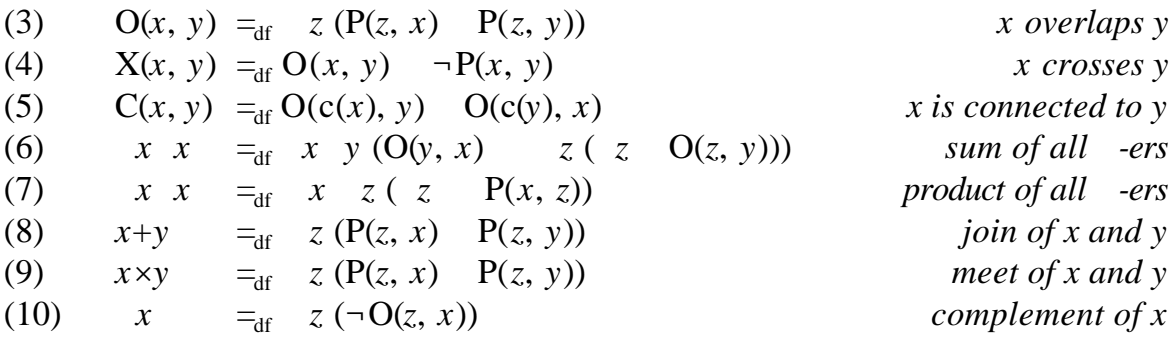

In addition to the these basic mereotopological notions, the following notions are introduced in [7] with an eye to their use in characterizing event domains:

(11) $\mathrm{D}(x)=_{\mathrm{df}} \exists y \exists z(y+z=\sim x \wedge \neg \mathrm{C}(y, z))$

(12) $\quad \mathrm{d}(x)={ }_{\mathrm{df}} \pi z(\mathrm{D}(z) \wedge \mathrm{P}(x, z))$

(13) $\mathrm{S}(x, y, z)=_{\mathrm{df}} y+z=\sim \mathrm{d}(x)$

$x$ is a divisor divisor of $x$ $x$ separates $y$ and $z$

Intuitively, divisors are distinguished events splitting their complement (the rest of history) into two disconnected parts. The idea is that a divisor comprises all that happens during certain period of time, so that events on one side of it count as past events and those on the other side as future events. On this basis, the construction proceeds as follows.

DEFINITION 1. An event structure is an ordered pair $\langle E, \delta\rangle$, where $E$ is a non empty domain and $\delta$ a subset of $E$ satisfying the following conditions for all $x, y \in E$ :

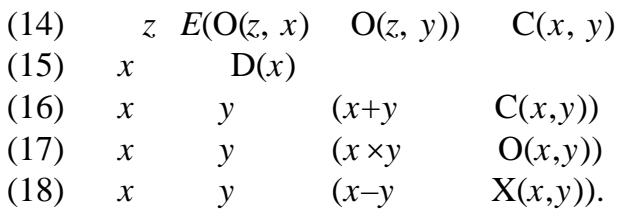

Intuitively, (14) says that $E$ is topologically self-connected and (15)-(18) that $\delta$ is a exhaustive set of "coherent" divisors in $E$ (i.e., divisors which cut history into a series of "parallel", mutually disjoint slices).

P. Bouquet, P. Brezillon, and L. Serafini (eds.), Modeling and Using Context. Proceedings of the Second International and Interdisciplinary Conference, Berlin and Heidelberg: Springer-Verlag, 1999, pp. 507-510. 
DEFINITION 2. An oriented event structure is a triple $\langle E, \delta, a\rangle$, where $\langle E, \delta\rangle$ is an event structure and $a$ is a distinguished element of $E$ such that

$$
\exists x \exists y(\mathrm{~S}(x, a, y)) .
$$

Intuitively, $a$ is an "anchor" element relative to which every other event can be positioned on the assumption that $a$ covers one of the two sides (intuitively, either the past or the future) of some event $x$. The positioning is obtained via the following:

$$
\begin{aligned}
& f(x)={ }_{\mathrm{df}} 1 z \exists y(\mathrm{~S}(z, x, y) \wedge(\mathrm{O}(x, a) \rightarrow \mathrm{P}(z, a)) \wedge(\neg \mathrm{O}(x, a) \rightarrow \mathrm{P}(a, z))) \\
& f^{\prime}(x)={ }_{\mathrm{df}} \sigma z(\mathrm{P}(x, f(z))) .
\end{aligned}
$$

(This effectively amounts to defining $f$ and $f^{\prime}$ as a pair of Galois connections.) We then just stipulate that $a$ represents the past. That is, we treat $f$ as a function of temporal orientation associating each event with the totality of events that precede it; correspondingly, we treat $f^{\prime}$ as a function associating each event with the events that follow it. This allows us to introduce a relation of temporal precedence by definition:

(22) $\left.\quad \mathrm{TP}(x, y)=_{\mathrm{df}} \mathrm{P}(x, f(y))\right)$

DEFINITION 3. A refinement event structure is a triple $\left\langle E,\left\{\delta_{i}: i \in I\right\}, a\right\rangle$ such that (i) for each $i \in I,\left\langle E, \delta_{i}, a\right\rangle$ is an oriented event structure, and (ii) the family $\left\{\delta_{i}: i \in I\right\}$ is closed under meet, i.e., for all $x, y \in E$ and all $i, j \in I$ there exists some $k \in I$ such that

$$
x \in \delta_{i} \wedge y \in \delta_{j} \rightarrow x \times y \in \delta_{k} .
$$

Thus, refinement structures involve not just one divisors class $\delta$, but an entire family of such classes. And condition (23) guarantees coherence among these classes, so that it is only the granularity that may change from one class to another. Indeed, the $\delta$ 's can be partially ordered in a natural way: a divisors class is a refinement of another iff the former draws at least the same temporal distinctions as the latter (if not more):

$$
\delta_{i} \S_{j}=x\left(x \in \delta_{i} \rightarrow \exists y\left(y \in \delta_{j} \wedge \mathrm{P}(y, x)\right)\right) .
$$

\section{Temporal Reference and Context Dependence}

Refinement structures allow us account for the effect of context on the granularity of temporal reference. With regard to the phenomenon exemplified in (1), the intuition that time is dense can be accommodated by adding the mereotopological counterpart of the usual axiom for dense linear orders on closed (or, equivalently, open) intervals:

$$
\mathrm{TP}(\mathrm{c}(x), \mathrm{c}(y)) \rightarrow \exists z(\mathrm{TP}(\mathrm{c}(x), \mathrm{c}(z)) \wedge \mathrm{TP}(\mathrm{c}(z), \mathrm{c}(y))) .
$$

More generally, in the context of a refinement structure $\left\langle E,\left\{\delta_{i}: i \in I\right\}, a\right\rangle$, this corresponds to assuming the following to hold for relevant $i \in I$ :

$$
\mathrm{P}\left(\mathrm{c}(x), f_{i}(\mathrm{c}(y))\right) \rightarrow \exists z\left(\mathrm{P}\left(\mathrm{c}(x), f_{i}(\mathrm{c}(z))\right) \wedge \mathrm{P}\left(\mathrm{c}(z), f_{i}(\mathrm{c}(y))\right)\right) .
$$

However this does not fully capture the idea behind (1). The interesting question is what kind of divisors are presupposed by the underlying unlimited refining process. Clearly they must be infinite in number (which in turn presupposes that the domain $E$

P. Bouquet, P. Brezillon, and L. Serafini (eds.), Modeling and Using Context. Proceedings of the Second International and Interdisciplinary Conference, Berlin and Heidelberg: Springer-Verlag, 1999, pp. 507-510. 
must have infinite cardinality). But, more importantly, they cannot include a minimal element (with respect to the ordering $\lesssim$ This amounts to the following requirement:

(27) For every $i \in I$ there exists $j \in I$ such that $\delta_{i} \S_{j}$ but not $\delta_{j} \S_{i}$.

This entails that divisors must themselves be infinitely divisible, i.e., there can be no absolute punctual events.

Consider now to the phenomenon exemplified in (2): we can discretize time at will. Intuitively, punctual events are instantaneous, i.e., do not extend over any time interval: they are located in time but do not take up time. Within the present setting, this does not amount to a requirement of mereological atomicity: what counts as instantaneous, as opposed to extended in time, depends entirely on the relevant $\delta_{i}$. For divisors not only provide the basis for temporal orientation but, in a sense, also for temporal measurement [4]. To see this, define the notion of a minimal divisor relative to a divisors class $\delta_{i}$ :

$$
\mathrm{M}_{i}(x)=_{\mathrm{df}} x \in \delta_{i} \wedge \forall y\left(\mathrm{P}(y, x) \rightarrow \neg y \in \delta_{i}\right) .
$$

In [8] we have shown that the fundamental properties characterizing punctual events according to Kamp [6] hold of minimal—and only minimal—divisors. Accordingly, we can define a punctual events, relative to the granularity set by $\delta_{i}$, to be exactly those events whose divisors are minimal relative to $\delta_{i}$ :

$$
\mathrm{PE}_{i}(x)={ }_{\mathrm{df}} \mathrm{M}_{i}(\mathrm{~d}(x)) .
$$

Thus, punctual events are not merely-and not necessarily-atomic events, i.e., events with no proper parts (although of course every atomic event is punctual, regardless of $\delta_{i}$ ). Rather, they are events whose internal structure is irrelevant for the purpose of temporal distinctions. By changing $\delta_{i}$, events previously treated as punctual may become non-punctual, in that their internal temporal structure is made available, and vice versa. This notion of "change" is purely metalinguistic if we focus on plain structures. But refinement structures can accommodate this variability directly.

\section{References}

1. Bach, E., 1986, 'The Algebra of Events', Linguistics and Philosophy 9: 5-16.

2. Casati, R., and Varzi, A. C., 1999, Parts and Places: The Structures of Spatial Representation, Cambridge, MA: MIT Press (Bradford Books).

3. Davidson, D., 1967, 'The Logical Form of Action Sentences', in N. Rescher (ed.), The Logic of Decision and Action, Pittsburgh: University of Pittsburgh Press, 81-95.

4. Giorgi, A., and F. Pianesi, 1997, Tense and Aspect: From Semantics to Morphosyntax, New York: Oxford University Press.

5. Kamp, H., 1979, 'Events, Instants, and Temporal Reference', in R. Bäuerle et al. (eds.), Semantics from Different Points of View, Berlin: Springer-Verlag, 376-417.

6. Pianesi, F. and A. C. Varzi, 1994, 'Mereo-Topological Construction of Time from Events', in A. Cohn (ed.), Proceedings ECAI-94, Chichester: Wiley \& Sons, 396-400.

7. Pianesi, F. and A. C. Varzi, 1996a, 'Events, Topology, and Temporal Relations', The Monist 78: 89-116.

8. Pianesi, F. and A. C. Varzi, 1996b, 'Refining Temporal Reference in Event Structures', Notre Dame Journal of Formal Logic 37: 71-83.

P. Bouquet, P. Brezillon, and L. Serafini (eds.), Modeling and Using Context. Proceedings of the Second International and Interdisciplinary Conference, Berlin and Heidelberg: Springer-Verlag, 1999, pp. 507-510. 